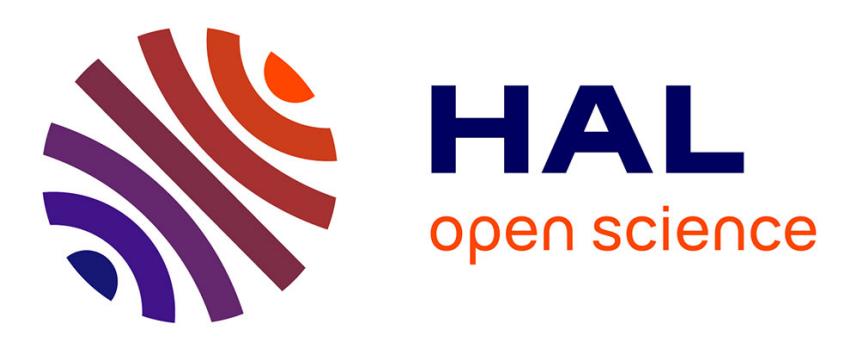

\title{
Induction Machines Fault Detection Based on Subspace Spectral Estimation
}

Youness Trachi, El Houssin El Bouchikhi, Vincent V. Choqueuse, Mohamed Benbouzid

\section{- To cite this version:}

Youness Trachi, El Houssin El Bouchikhi, Vincent V. Choqueuse, Mohamed Benbouzid. Induction Machines Fault Detection Based on Subspace Spectral Estimation. IEEE Transactions on Industrial Electronics, 2016, 63 (9), 10.1109/TIE.2016.2570741 . hal-01356659

\section{HAL Id: hal-01356659 https://hal.science/hal-01356659}

Submitted on 26 Aug 2016

HAL is a multi-disciplinary open access archive for the deposit and dissemination of scientific research documents, whether they are published or not. The documents may come from teaching and research institutions in France or abroad, or from public or private research centers.
L'archive ouverte pluridisciplinaire HAL, est destinée au dépôt et à la diffusion de documents scientifiques de niveau recherche, publiés ou non, émanant des établissements d'enseignement et de recherche français ou étrangers, des laboratoires publics ou privés. 


\title{
Induction Machines Fault Detection Based on Subspace Spectral Estimation
}

\author{
Youness Trachi, Elhoussin Elbouchikhi, Vincent Choqueuse, Member, IEEE, and M. Benbouzid, Senior \\ Member, IEEE.
}

\begin{abstract}
The main objective of this paper is to detect faults in induction machines using a condition monitoring architecture based on stator current measurements. Two types of fault are considered: bearing and broken rotor bars faults. The proposed architecture is based on high-resolution spectral analysis techniques also known as subspace techniques. These frequency estimation techniques allow to separate frequency components including frequencies close to the fundamental one. These frequencies correspond to fault sensitive frequencies. Once frequencies are estimated, their corresponding amplitudes are obtained by using the Least Squares Estimator (LSE). Then, a fault severity criterion is derived from the amplitude estimates. The proposed methods were tested using experimental stator current signals issued from two induction motors with the considered faults. The experimental results show that the proposed architecture has the ability to efficiently and cost-effectively detect faults and identify their severity.

Index Terms-Induction machine, bearing faults, broken rotor bar faults, stator current analysis, subspace techniques, RootMUSIC, ESPRIT, fault severity detection.
\end{abstract}

\section{INTRODUCTION}

I NDUCTION machines are the most common electrical machines used in industry applications. The use of induction machines in electromechanical conversions is mainly due to their advantages such as low cost, reliability, availability, and high robustness [1]. However, these electromechanical devices are susceptible to many types of faults. The main induction machine fault types studied in the literature are generally classified into electrical and mechanical faults [2]-[4]. The most common electrical faults that could appear are open or short circuit in motor windings (mainly due to winding insulation failure), wrong connection of windings, high resistance contact to conductor, and wrong or unstable ground. Besides, mechanical faults include: broken rotor bars, cracked end-rings, bent shaft, bolt loosening, bearing failure, gearbox failure, and air-gap irregularity [5]. Induction machine faults can cause shutdowns, components degradation, vibrations, and expensive maintenance cost. Consequently, developing noninvasive condition monitoring and fault diagnosis techniques seems an

Manuscript received October 14, 2015; revised February 16, 2016; accepted March 31, 2016.

Youness Trachi, Vincent Choqueuse and Mohamed Benbouzid are with the University of Brest, FRE CNRS 3744 IRDL - Institut de Recherche Dupuy de Lôme, Rue de Kergoat, CS 93837, 29238 Brest Cedex 03, France (e-mail: Youness.Trachi@univ-brest.fr, Vincent.Choqueuse@univbrest.fr, Mohamed.Benbouzid@univ-brest.fr). Mohamed Benbouzid is also with the Shanghai Maritime University, 201306 Shanghai, China.

Elhoussin Elbouchikhi is with the ISEN Brest, FRE CNRS 3744 IRDL Institut de Recherche Dupuy de Lôme, 20, Rue Cuirassé Bretagne, 29200 Brest, France (email: elbouchikhi@isen-bretagne.fr). attractive solution to reduce operating and maintenance costs. Most methods for condition monitoring and fault detection of induction machines can be classified into the following categories: vibration monitoring, temperature monitoring, oil or debris analysis, acoustic emission monitoring, and current, voltage, or power monitoring [5]. These technologies require additional sensors and specific data acquisition devices to be implemented and are difficult to access during induction machine operation. Under fault condition, stator current-based condition monitoring has received a great deal of attention in the recent years to develop a non-invasive, a lower-cost, and a reliable technology that fully exploit the benefits of induction machines condition monitoring [6]-[8]. The use of the stator current presents advantages such as its easy-access, the ease of implementation, the information richness, and the ability to detect electrical and mechanical faults. In fact, stator current analysis-based condition monitoring do not require an additional sensors or data acquisition devices. A general reviews of induction machines monitoring and faults diagnosis techniques are available in [2], [9]-[11].

It has been demonstrated that faults introduce additional frequency components in the stator current signal. Therefore, several advanced spectral estimation techniques using stator currents have been proposed to detect these fault frequencies. There are mainly two types of power spectrum estimation (PSE): parametric and nonparametric methods. Nonparametric methods estimate the power spectrum directly from the measurements. These methods constitute the classical means for Power Spectral Density (PSD) estimation. These methods include the conventional periodogram and its extensions [12]-[14]. However, these techniques have several drawbacks since their frequency resolution is limited and long data measurements are required. To overcome these issues, many different parametric techniques have been developed for PSE. These parametric methods for PSE are based on parametric models to represent the signal and then to estimate model parameters from the available signal data. Parametric methods for spectral estimation are divided into two classes: parametric for continuous spectra and parametric for line spectra [15]. The parametric techniques for continuous spectra include the linear prediction techniques. The linear prediction techniques contain several algorithms like the Prony and Pisarenko methods. These methods are specifically designed for continuous PSD, where the frequency content does not vary abruptly. Unfortunately, these methods are not suited for fault frequency estimation because the fault signature introduces specific frequencies close to the fundamental frequency. The paramet- 
ric techniques for line spectra include Maximum Likelihood Estimator (MLE) and subspace techniques called also highresolution techniques. The MLE is an asymptotically optimal estimator but it requires a multidimensional grid search that increases drastically when the model order increases [16]. The applications of MLE-based spectral estimations for machine fault detection are available in [17], [18]. Indeed, in [17], [18], a model order and spectral estimations based on MLE are proposed to detect induction machine fault frequency signatures. In stator current analysis, subspace techniques have been proposed to avoid the computational complexity inherent to multidimensional optimization of MLE [4], [19]-[26]. The subspace techniques include the MUSIC (MUltiple SIgnal Classification) and ESPRIT (Estimation of Signal Parameters via Rotational Invariance Techniques) approaches.

In induction machine condition monitoring, the problem of frequency estimation using subspace techniques has received a lot of attention in the electrical engineering community. Motors faults detection technique using high-resolution spectral analysis has been proposed in [20]. In [20], the authors propose to use the Spectral-MUSIC or Root-MUSIC for frequency estimation. An application of high-resolution spectral analysis for identifying multiple combined faults in induction motors can be found in [26]. The major contribution of [26] is the development of a condition-monitoring strategy that allows accurate and reliable assessments of the presence of specific fault conditions in induction motors with single or multiple combined faults. This proposed condition monitoring strategy is based on the combination between a finite impulse response filter bank to separate the original current and vibration signals into different fault-related bandwidths and the Spectral-MUSIC to detect frequencies of the stator current. This methodology can detect two faults: bearing and broken rotor bars faults. A fusion between two techniques: the Complete Ensemble Empirical Mode Decomposition (CEEMD) and the MUSIC is proposed in [27]. In this case, the proposed methodology allows identifying time evolution of the faulty frequencies in start-up and steady-state regimes from the short data record signal buried in noise, as it is the case of inverter-fed induction motors. Another technique has been proposed in [28] to detect incipient broken rotor bar in induction motors using high-resolution spectral analysis based on the start-up current analysis. This technique is based on the short-time MUSIC algorithm that provides high-resolution and the time-frequency pseudo-representation. The proposed methods can graphically show the physical effect of a broken or partially-broken rotor bar. A modified version of the MUSIC algorithm has been developed in [4] to estimate the stator current spectrum. In [4], a fault detection criterion is proposed to detect faults. This criterion does not take into account the harmonic structure of the stator current. An application of the ESPRIT and the Simulated Annealing Algorithm (SAA) has been proposed to detect broken rotor bar fault in induction motors with shorttime measurement data in [25]. The proposed techniques in [25] can correctly identify the parameters of the broken rotor bars characteristic components with short-time measurement data. Another fusion between two techniques: the Hilbert transform and the ESPRIT for detecting rotor fault in induction motors at low slip has been proposed in [24]. This fusion combines two main characteristics: ability to avoid spectral leakage and to achieve high-frequency resolution even with a short measurement time.

Another application of high-resolution frequency estimation method for three-phase induction machine fault detection has been suggested in [29]. The proposed Zoom-MUSIC in [29] is used to detect broken rotor bars fault using spectrum analysis in induction machine under different loads and in steady-state condition. The proposed technique in [29] allows reducing the computational complexity focusing on frequencies close to the fundamental frequency. In this case, the model order is obtained by the Frequency Signal Dimension Order (FSDO) estimator proposed in [30]. The application of high-resolution parameter estimation method to identify broken rotor bar faults in induction motors has been proposed recently in [21]. The authors in [21] propose two algorithms Zoom-MUSIC and Zoom-ESPRIT to estimate frequencies in order to reduce the long computation times required by classical subspace techniques from short data signals with low Signal-to-Noise Ratio (SNR). In this case, the model order is obtained by the FSDO estimator proposed in [31]. In [21], a fault detection criterion based on false alarm and detection probabilities is proposed to detect faults. A comparative study and the evaluation of various condition monitoring methods used for induction machines, with the aim of early detection of one partially-broken rotor bar by steady-state current spectrum analysis and different supply conditions is proposed in [32]. The techniques considered in this study are the Fast Fourier transform (FFT), Wavelet plus FFT, MUSIC, Empirical Mode Decomposition (EMD) plus FFT, and EMD associated with MUSIC. Broken rotor bar detection in variable speed drivefed induction motors at start-up by high-resolution spectral analysis has been proposed in [33]. In this case, the timefrequency spectrum is able to graphically show a different pattern for the healthy and faulty conditions. Finally, a stator current analysis by subspace methods for fault detection in induction machines has been proposed in [19]. Two subspace techniques: Root-MUSIC and ESPRIT are presented and a fault severity criterion with a fault severity criterion.

This paper focuses on two induction machines faults: bearing and broken rotor bar faults. To detect these faults, we propose to use advanced signal processing and statistical analysis techniques to estimate a group of signal parameters that are assumed to be stationary. We present frequency estimators based on subspace techniques such as MUSIC and ESPRIT. These methods require the exact knowledge of either the number of sinusoids in the data. In order to detect the model order, we propose to use information criteria. A Fault Severity Criterion (FSC) is also proposed to measure the degree of the considered faults based on the amplitude evaluation corresponding to estimated frequencies. These amplitudes are obtained by the Least Squares Estimator (LSE).

The main contributions of this paper, compared to previously published and above-discussed ones, can be summarized by:

- Many papers assume the model order to be known [20], [25], [26], [28], [32]. However, the performances 
of subspace techniques completely degrade when using a wrong model order. Consequently, a model order estimation step is required to estimate faulty frequencies appearing in the stator current spectrum based on subspace spectral estimation. In [19], [21], [29], several authors have proposed to use model order estimation techniques based on the eigenvalue decomposition of the covariance matrix proposed in [30], [31]. However, these eigenvalue-based techniques are general and do not take into account the particular structure of the signal subspace. From an estimation viewpoint, the particular structure of the signal subspace offers opportunity to improve the estimation of the model order, which is of main concern in spectral estimation. To fulfill this problem, we propose to use the model order estimator given in [34] that associates the order-selection rule with the maximum likelihood method. In this paper, we have also demonstrated using Monte-Carlo trials that the best model order selection for the considered stator current structure is the Bayesian Information Criterion (BIC).

- A new Fault Severity Criterion (FSC) is proposed to determine the state of the studied machines. Moreover, the proposed FSC allows to detect the faults degree even in the presence of harmonic components. The main advantages of the proposed fault detection criterion is its low computational complexity and its ability to automatically detect faults without the need of an expert for interpreting the stator current spectrum.

The remainder of the paper is organized as follows. Section II presents the problem formulation of stator current based condition monitoring. Section III gives the estimation of stator current model parameters. Section IV provides the proposed fault severity criterion and the condition monitoring architecture. Section V shows the experimental results for the considered faults. Section VI concludes this paper.

\section{Problem Formulation}

In this section, we present the stator current model under bearing faults and broken rotor bar faults.

\section{A. Stator Current Model}

Stator current analysis using advanced signal processing techniques needs to select a good model in order to approximate the signal. The stator current signal model is based on the following assumptions. The received signal is modeled as a sum of $L$ exponential components in noise. The phases of the exponential components are independent and uniformly distributed on the interval $[-\pi, \pi[$.

In practice, a noise component is added to take into account the measurement errors. We assume the noise as a white Gaussian noise with zero-mean and variance $\sigma^{2}$. This assumption is motivated by the following reasons:

- The Gaussian noise assumption leads to minimize the worst-case asymptotic Cramer-Rao Bound (CRB) [35];

- The Minimum Variance Unbiased (MVU) estimator is equivalent to the mean LSE when the noise is white Gaussian [16];
- The sum of a sufficiently large number of independent and identically random variables are approximately Gaussian distributed (Central Limit Theorem) [36].

According to the above-mentioned assumptions, the induction machine stator current in presence of faults can be described by the following model

$$
x[n]=\sum_{k=0}^{L-1} a_{k} e^{j\left(2 \pi f_{k} \times \frac{n}{F_{s}}+\phi_{k}\right)}+b[n]
$$

where $x[n]$ denotes the stator current samples, $b[n] \sim$ $\mathcal{N}_{c}\left(0, \sigma^{2}\right)$ is a white Gaussian noise, $L$ represents the model order, $F_{s}$ is the sampling frequency, $a_{k}, f_{k}$, and $\phi_{k}$ are amplitude, frequency, and initial phase of the $k^{\text {th }}$ component, respectively.

At time $n=0,1,2,3, \ldots, N$, the observed stator current vector $\mathbf{x}$, defined as $\mathbf{x}=\left[\begin{array}{lll}x(n) & \ldots & x(n+M-1)\end{array}\right]^{T}$, can be expressed as

$$
\mathbf{x}=\mathbf{A}(\theta) \mathbf{s}+\mathbf{b}
$$

where

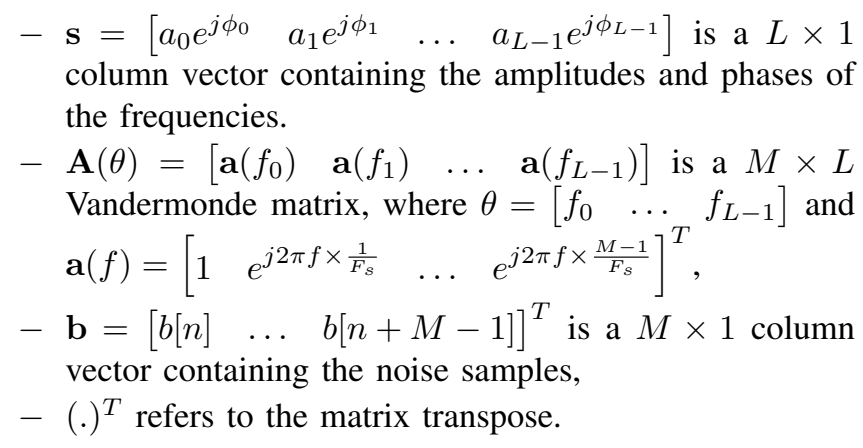

\section{B. Stator Current Frequency Model}

In stationary environment, the stator current spectrum of induction machines without faults contains only the fundamental frequency and harmonics. In the international standard IEC 038 of electrical engineering, frequencies of the stator current spectrum may have a small variation up to $1 \%$ of the fundamental frequency value [37]. In case of fault presence, the spectrum also contains frequencies called fault frequencies according to the following relationship [1]

$$
f_{f}=f_{s} \pm k f_{c},
$$

where $f_{s}$ is the supply fundamental frequency, $f_{c}$ is the fault characteristic frequency, and $k \in \mathbb{N}^{*}$. In this paper, we consider two faults: bearing faults and Broken Rotor Bar (BRB) faults. The exact value of the characteristic frequency with respect to the fault parameters are given in [2].

The purpose of the next section is to present techniques used to estimate model parameters given in (1).

\section{Parametric Spectral Analysis}

In this paper, condition monitoring based on stator current processing needs to estimate values of unknown stator current model parameters. A three steps process is therefore needed: frequency estimation, amplitude complex estimation, and model order selection. 


\section{A. Parameter Estimation}

The objective is to estimate model parameters from stator current measurements. The model parameters estimates can be obtained using the Nonlinear Least Squares Estimator (NLSE) [15], [16]. These estimations are obtained by the squared deviations minimization between stator current measurements and the assumed stationary model [15], [16]. These estimation problems using NLSE can be expressed by the following cost function

$$
\widehat{\boldsymbol{\Omega}}=\underset{\boldsymbol{\Omega}}{\operatorname{argmin}}\|\mathbf{x}-\mathbf{A}(\theta) \mathbf{s}\|^{2},
$$

where $\boldsymbol{\Omega}=\left[\begin{array}{ll}\theta & \mathbf{s}\end{array}\right]$ denotes the unknown stator current model parameters. Note that, when the noise is assumed to be white and Gaussian, this estimator corresponds to the MLE. The main drawback of the NLSE is its computation cost since the estimation of the frequencies requires the maximization of a multidimensional and multimodal cost function [17], [18].

In this paper, as a first step, it is assumed that the model order is known. Using this assumption, we propose to use subspace techniques for frequency estimation. Once frequencies are estimated, we use linear least squares estimator to estimate the vector of complex amplitudes s. Finally, we also propose a technique for the estimation of $L$ when the model order is unknown.

\section{B. Frequency Estimation}

To estimate frequencies, the natural estimator is the NLSE [15]. To avoid the computational complexity inherent to multidimensional optimization, we resort to subspace techniques. Subspace estimation techniques are based on the eigendecomposition of the covariance matrix $\mathbf{R}_{x}$. This decomposition permits to separate two distinct subspaces, the signal subspace and the noise subspace. Thus, the covariance matrix can be written as a sum of the signal and the noise covariance matrices

$$
\mathbf{R}_{x}=\mathbf{R}_{s}+\mathbf{R}_{n}=\left[\begin{array}{ll}
\mathbf{S} & \mathbf{G}
\end{array}\right]\left[\begin{array}{cc}
\boldsymbol{\Lambda}_{s} & 0 \\
0 & \boldsymbol{\Lambda}_{n}
\end{array}\right]\left[\begin{array}{ll}
\mathbf{S} & \mathbf{G}
\end{array}\right]^{H},
$$

where $\mathbf{R}_{s}$ denotes the signal covariance matrix, $\mathbf{R}_{n}$ is the noise covariance matrix, $\boldsymbol{\Lambda}_{s}=\operatorname{diag}\left[\begin{array}{lll}\lambda_{1} & \ldots & \lambda_{L}\end{array}\right]$ and $\boldsymbol{\Lambda}_{n}=\operatorname{diag}\left[\begin{array}{lll}\lambda_{L+1} & \ldots & \lambda_{M}\end{array}\right]$ are diagonal matrices containing eigenvalues of the signal and the noise subspaces arranged in descending order, respectively ( $\mathbf{S}$ and $\mathbf{G}$ are the associated orthonormal eigenvectors, respectively). In practice, the theoretical covariance matrix $\mathbf{R}_{x}$ and its eigendecomposition are not known but can be estimated from observations as follows

$$
\widehat{\mathbf{R}}_{x}(n)=\frac{1}{G} \sum_{n=1}^{G} \mathbf{x}(n) \mathbf{x}^{H}(n),
$$

where $\mathbf{x}=\left[\begin{array}{lll}x(n) & \ldots & x(n+M-1)\end{array}\right]^{T}$ has length $M$. Since we have $N$ observations of $x[n], G=N-M+1$. In the following subsections, we describe two subspace techniques: MUSIC estimators based on the noise subspace and ESPRIT estimators based on the signal subspace.
1) MUSIC Estimators: The MUSIC frequency estimates are determined as the minimizing arguments of the following cost function

$$
f(\theta)=\operatorname{Tr}\left(\mathbf{A}(\theta) \mathbf{A}^{H}(\theta) \mathbf{G G}^{H}\right) .
$$

If we replace the matrix expression of $\mathbf{A}(\theta)$ in (7), we can write

$$
\widehat{\theta}=\arg \min _{\{f\}}\left\|\mathbf{a}^{H}(f) \widehat{\mathbf{G}}\right\|_{F}^{2},
$$

where $\|\cdot\|_{F}$ denotes the Frobenius norm. There exist two kinds of MUSIC implementations, Spectral-MUSIC and RootMUSIC [38].

The Spectral-MUSIC finds the minimizing arguments of $f(\theta)$ by a one-dimensional search algorithm as follows

$$
\widehat{\theta}=\arg \max _{\{f\}} \frac{1}{\left\|\mathbf{a}^{H}(f) \widehat{\mathbf{G}}\right\|_{F}^{2}},
$$

Frequency estimation is obtained by finding the $L$-highest local maxima of the (9) called pseudo-spectrum function.

The Root-MUSIC finds the minimizing arguments of $f(\theta)$ by polynomial rooting to avoid searching for peaks [38], [39]. This estimator converts this pseudo-spectrum function into a polynomial representation that is given by

$$
Q(z)=\mathbf{a}^{H}\left(\frac{1}{z^{*}}\right) \mathbf{G G}^{H} \mathbf{a}(z),
$$

where $\mathbf{a}(z)=\left[\begin{array}{llll}1 & z & \ldots & z^{M-1}\end{array}\right]^{T}$ is a column vector and $z=e^{\frac{j 2 \pi f}{F_{s}}}$. The Root-MUSIC algorithm requires to finds the roots of the complex polynomial function $z^{M-1} Q(z)$ for frequency estimation

$$
Q(z)=z^{-(M-1)}\left[\begin{array}{llll}
z^{M-1} & \ldots & z & 1
\end{array}\right] \mathbf{G G}^{H}\left[\begin{array}{c}
1 \\
z \\
\vdots \\
z^{M-1}
\end{array}\right] .
$$

Thus, the Root-MUSIC algorithm finds the roots of $\tilde{Q}(z)$, that is a complex polynomial function given by

$$
Q(z)=z^{-(M-1)} \tilde{Q}(z),
$$

where $\tilde{Q}(z)$ is a $2(M-1)$ degree polynomial in $z$ the roots of which come in pairs, since, by construction, if $z_{0}$ is a root, then $\frac{1}{z_{0}^{*}}$ is a root. Once the polynomial $\tilde{Q}(z)$ is obtained, the frequency estimation can be determined by calculating the $2(M-1)$ roots of $\tilde{Q}(z)$, then keeping the $L$ stable roots that are closest to the unit circle. The relationship between $L$ roots and frequencies is given by

$$
\widehat{f}_{k}=\frac{\arg \left(\widehat{z}_{k}\right)}{2 \pi} \times F_{s}
$$

where $\widehat{z}_{k}$ denotes the $k^{\text {th }}$ root of $\tilde{Q}(z)$. Note that many programming language contain functions for root finding.

Root-MUSIC has the same asymptotic performance as Spectral-MUSIC [39]. Since the search procedure in SpectralMUSIC is replaced by solving the roots of a polynomial in Root-MUSIC, the computational cost is significantly reduced [38]. Thus, it is preferable to use only the Root-MUSIC estimator for practical applications. 
2) ESPRIT Estimators: The key element of ESPRIT is to use the rotational property between staggered subspaces for frequency estimation [40], [41]. Two extensions of ESPRIT methods are considered: Least-Squares (LS) ESPRIT and Total Least-Squares (TLS) ESPRIT.

Let $\mathbf{S}_{1}=\left[\begin{array}{ll}\mathbf{I}_{M-1} & 0\end{array}\right] \mathbf{S}$ and $\mathbf{S}_{2}=\left[\begin{array}{ll}0 & \mathbf{I}_{M-1}\end{array}\right] \mathbf{S}$ be unstaggered and staggered signal subspaces, respectively. In the LS ESPRIT, we estimate frequencies by using eigenvalues of $\boldsymbol{\Phi}_{L S}$ that are given by

$$
\mathbf{\Phi}_{L S}=\left(\mathbf{S}_{1}^{H} \mathbf{S}_{1}\right)^{-1} \mathbf{S}_{1}^{H} \mathbf{S}_{2} .
$$

This LS solution is obtained by minimizing the estimation error on $\mathbf{S}_{1}$. We can do better by using the TLS ESPRIT that is obtained by minimizing estimation errors on $\mathbf{S}_{1}$ and $\mathbf{S}_{2}$. In this technique, we estimate frequencies by using the Singular Value Decomposition (SVD) of $\left[\begin{array}{ll}\mathbf{S}_{1} & \mathbf{S}_{2}\end{array}\right]=\mathbf{L} \boldsymbol{\Sigma} \mathbf{V}^{H}$ [40], where $\mathbf{L}$ is a matrix of left singular vectors, $\boldsymbol{\Sigma}$ is a matrix consisting of singular values on the main diagonal ordered in descending magnitude, and $\mathbf{V}$ is a matrix of right singular vectors. The matrix $\mathbf{V}$ is an $(2 L \times 2 L)$ unitary matrix, which can partitioned into $(L \times L)$ quadrants according to

$$
\mathbf{V}=\left[\begin{array}{ll}
\mathbf{V}_{11} & \mathbf{V}_{12} \\
\mathbf{V}_{21} & \mathbf{V}_{22}
\end{array}\right]
$$

In the TLS solution, we estimate frequencies by using eigenvalues of $\boldsymbol{\Phi}_{T L S}$ that are given by

$$
\boldsymbol{\Phi}_{T L S}=-\mathbf{V}_{11} \mathbf{V}_{22}^{-1} .
$$

In practice, we can estimate signal frequencies using the following expression

$$
\widehat{f_{k}}=\frac{\arg \left(v_{k}\right)}{2 \pi} \times F_{s},
$$

where $v_{k}$ are eigenvalues of $\widehat{\boldsymbol{\Phi}}_{L S}$ or $\widehat{\boldsymbol{\Phi}}_{T L S}$.

TLS-ESPRIT involves slightly more computations but it is generally preferred over the LS-ESPRIT due to its statistical performance [40]. Thus, it is preferable to use the TLSESPRIT estimator for practical applications.

\section{Complex Amplitude Estimation}

A number of complex amplitude estimators are developed in the literature, including the Maximum Likelihood Estimator (MLE), Least Squares Estimator (LSE), and Weighted Least Squares Estimator (WLSE). A survey of amplitude estimation techniques for sinusoidal signals with known frequencies can be found in [42]. Once frequencies are estimated, we propose to use the LSE for complex amplitude estimation. This estimator is given by

$$
\widehat{\mathbf{s}}=\left(\mathbf{A}^{H}(\widehat{\theta}) \mathbf{A}(\widehat{\theta})\right)^{-1} \mathbf{A}^{H}(\widehat{\theta}) \mathbf{x} .
$$

Finally, amplitudes and phases can be obtained as

$$
\widehat{a}_{k}=\left|\widehat{s}_{k}\right|, \quad \widehat{\phi}_{k}=\arg \left(\widehat{s}_{k}\right),
$$

where $\widehat{s}_{k}$ the $k^{\text {th }}$ component of $\widehat{\mathbf{s}},|$.$| denotes the complex$ modulus, and $\arg ($.$) represents the argument.$

\section{Model Order Selection}

Parametric spectral estimation methods require the knowledge of the model order. The model order can be obtained by minimizing information criteria such as Akaike Information Criterion (AIC), Bayesian Information Criterion (BIC), and Generalized Information Criterion (GIC). A review of information criterion can be found in [34]. A comparison between the proposed information criteria is required to determine the best model order estimator. We compare between these criteria using Monte-Carlo trials. In this context, we have studied two parameters influence on the model order selection: The samples number and Signal-to-Noise Ratio (SNR).

The model order is obtained by minimizing the following information criteria

$$
\widehat{L}=\arg \min _{L} N \ln \left(\widehat{\sigma}_{L}^{2}\right)+n * \eta(L, N),
$$

where $N$ is the number of samples, $n=3 L+1, \eta(L, N)$ is a penalty coefficients, which depend on information criteria available in [34], and $\widehat{\sigma}_{L}^{2}$ denotes the noise variance given by

$$
\widehat{\sigma}_{L}^{2}=\frac{1}{N} \sum_{n=0}^{N}\left|x[n]-\sum_{k=0}^{L-1} \widehat{a}_{k} e^{j\left(2 \pi \widehat{f}_{k} \times \frac{n}{F_{s}}+\widehat{\phi}_{k}\right)}\right|^{2} .
$$

For noise variance estimation, we have used the TLS ESPRIT to estimate frequencies and LSE for amplitudes and phases estimations.

Figure 1 shows the samples number influence on the model order selection with $\sigma^{2}=0.1$. Figure 2 shows the SNR influence on the model order selection with $N=400$. These simulation examples are obtained with amplitudes and phases are randomly generated according to

$$
a_{k}=\left|d_{k}\right|+1, \quad \phi_{k}=\arg \left(d_{k}\right)
$$

where $d_{k}$ is the $k^{\text {th }}$ component of $\mathbf{d}$, which is a normally distributed pseudorandom numbers. Frequencies $f_{k}=50 \pm k \times 5$, and $k \in\{0,1, \ldots, 4\}$. The probabilities are expressed in $\%$ and are obtained for 2000 Monte Carlo trials. According to previous Figs. 1 and 2, the probability of model order detection increases when samples number or SNR increases. In this example, we observe that the best criterion for model order selection is the BIC technique. The penalty coefficients of BIC technique is given by

$$
\eta(L, N)=\ln (N) .
$$

\section{Proposed Condition Monitoring Architecture}

This section proposes a condition monitoring architecture of induction machines based on model parameters estimation of the stator current. A fault severity criterion is also proposed to measure the fault severity.

\section{A. Proposed Fault Severity Criterion}

The proposed fault severity criterion (FSC) is needed to measure the machine state and to detect the fault severity. This criterion is based on the evaluation of frequency component amplitudes obtained with (19). It is an extension of the 


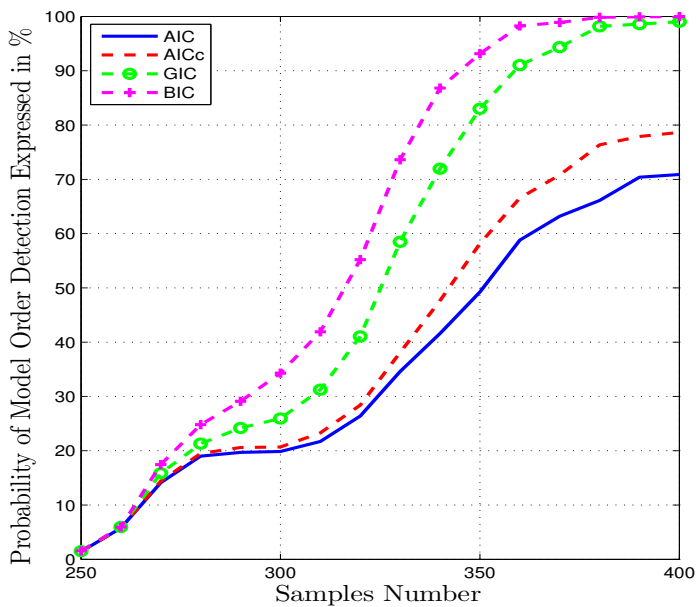

Fig. 1. Samples number influence on the model order selection with $\sigma^{2}=$ 0.1 .

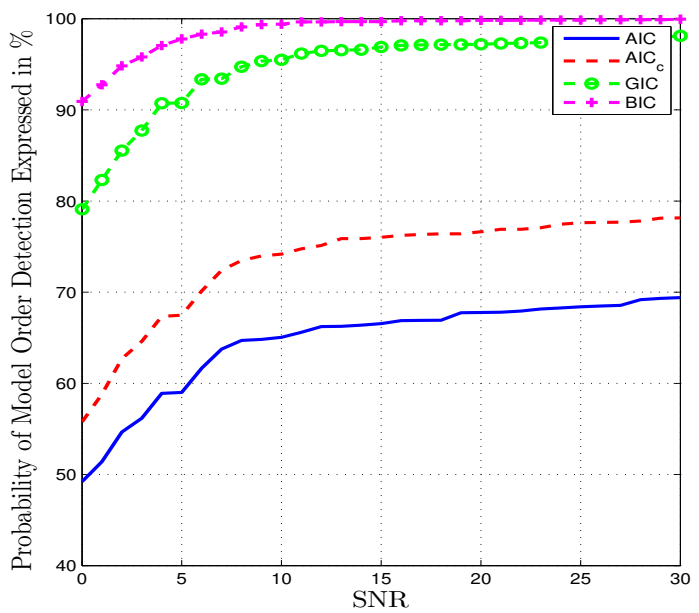

Fig. 2. SNR influence on the model order selection with $N=400$.

proposed criterion in [4]. The FSC is inspired from the total harmonic distortion (THD) of a signal, which is defined as the ratio of the sum of the powers of all harmonic components to the power of the fundamental frequency. Mathematically, the FSC depends on amplitudes $a_{k}$ and it can be expressed as

$$
C=\frac{\sum_{k \in \theta_{1}} \widehat{a}_{k}^{2}}{\sum_{l \in \theta_{2}} \widehat{a}_{l}^{2}},
$$

where $\theta_{1}$ corresponds to the integers $k$ that belong to $[0, L-1]$ for which $\left|\widehat{f}_{k}-n f_{s}\right|>\Delta f(n \in \mathbb{N}), \theta_{2}$ corresponds to the integers $l$ that belong to $[0, L-1]$ for which $\left|\widehat{f}_{l}-n f_{s}\right|<\Delta f$ $(n \in \mathbb{N})$, and $\Delta f=10^{-2} f_{s}$ is the authorized variation of frequency values according to Standard IEC 038 [37].

The proposed criterion is theoretically equal to zero for healthy induction machines and increases for a faulty case. In practice, the FSC value $C$ gives the induction machine state, which is compared with to the FSC value for healthy condition. Performance of this proposed criterion depends on the performances of model order, frequency, and amplitude estimators. This criterion can be explained by the algorithm described in Algo. 1. In this algorithm, 4.7 denotes the round function. This algorithm is characterized by its ease of implementation.

The purpose of the proposed algorithm is to calculate the FSC value that determines the state of the studied machine. Three steps are needed: model order selection, frequency estimation, and amplitude estimation. Once amplitudes are determined, we can compute the FSC ratio value. The denominator of this ratio contains the sum of squared amplitudes corresponding to the fundamental frequency and harmonics. The numerator contains the sum of the squared amplitudes corresponding to others frequencies. Therefore, a frequency evaluation is needed while respecting the authorized variation according to standard IEC 038.

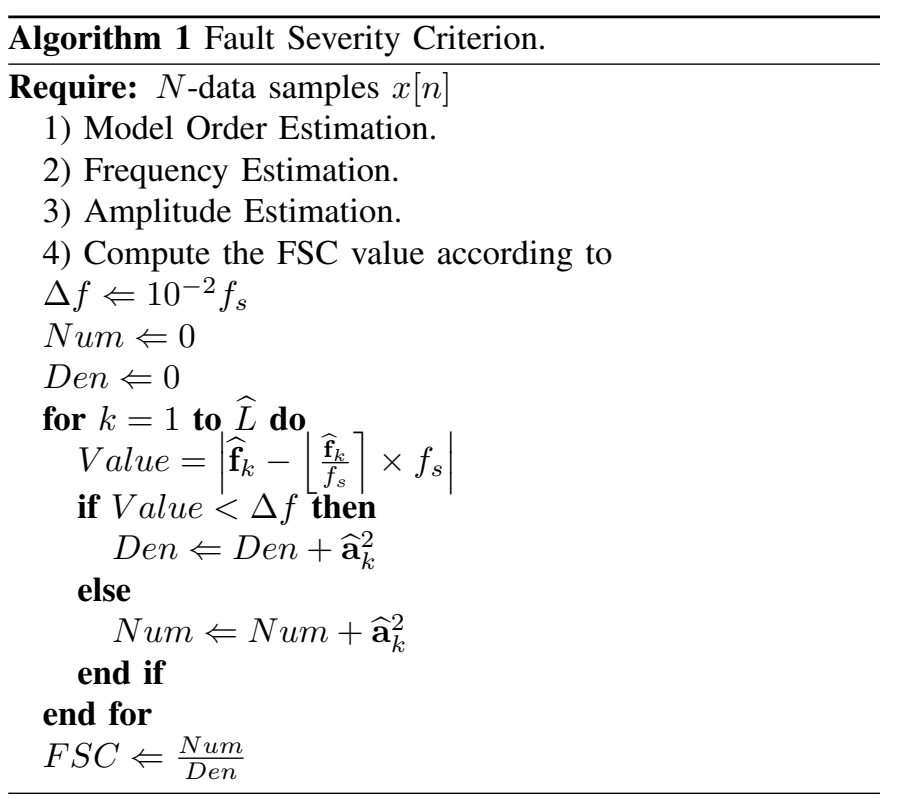

\section{B. Proposed Architecture}

The proposed condition monitoring architecture for induction machines is given by Fig. 3. It is based on the use of advanced signal processing techniques to estimate parameters of the stator current. These parameters are exploited to determine the value of the proposed FSC. This value allows evaluating the induction machine state. One of the valuable advantages of the proposed architecture is to detect faults in presence of signal parameters.

\section{EXPERIMENTAL RESUlts}

The efficiency of the proposed approach is evaluated on experimental stator currents issued from an induction machine with bearing faults and broken rotor bars. Several fault degrees are considered to evaluate the ability of the proposed criterion to track fault severity. A comparison between currents spectrum for healthy and faulty machines, allows distinguishing the frequency components that belong to the induction machine faults. 


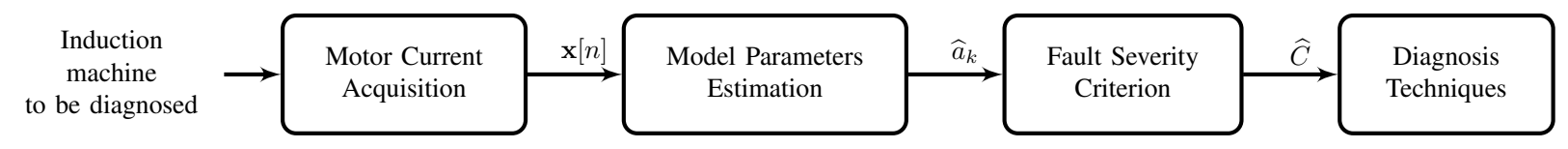

Fig. 3. Proposed condition monitoring architecture of induction machines.

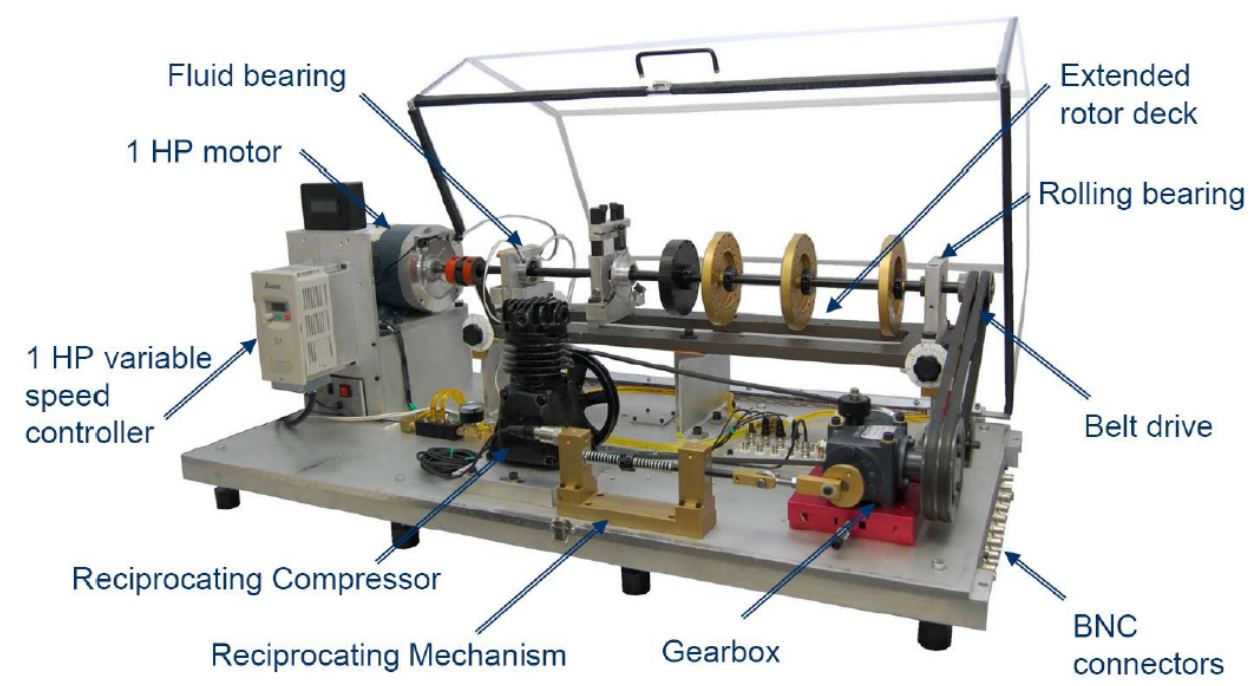

Fig. 4. Machinery fault simulator.

TABLE I

BEARING FAULT SEVERITY VERSUS HOLE DIAMETER.

\begin{tabular}{|c||c|c|c|c|c|}
\hline $\begin{array}{c}\text { Fault } \\
\text { severity }\end{array}$ & 1 & 2 & 3 & 4 & 5 \\
\hline $\begin{array}{c}\text { Bearing hole } \\
\text { diameter (inches) }\end{array}$ & 0.007 & 0.014 & 0.02 & 0.03 & 0.04 \\
\hline
\end{tabular}

\section{A. Experimental Setup Description}

Two induction motors have been studied. The first induction machine with bearing faults. In this case, the machine under test is a $230 / 400 \mathrm{~V}, 0.75-\mathrm{kW}$, three phases induction motor with the number of pole pairs $p=1$ and $2780 \mathrm{rpm}$ rated speed. The induction machine has two 6204-2 ZR type bearings (single row and deep groove ball bearings) with the following parameters: outside diameter is $47 \mathrm{~mm}$, inside diameter is $20 \mathrm{~mm}$, and pitch diameter $\mathrm{D}$ is $31,85 \mathrm{~mm}$. Bearings have 8 balls with an approximate diameter d of $12 \mathrm{~mm}$. Bearing faults are obtained by drilling holes of several diameters in the inner raceway (faults ranging from 0.007 inches $(0.178$ $\mathrm{mm})$ in diameter to 0.040 inches $(1.016 \mathrm{~mm})$ as it can be seen in Table I). The stator currents acquisition is performed by a 24 bits acquisition card with $10 \mathrm{kHz}$ sampling frequency.

The second induction machine is a $230 / 400 \mathrm{~V}, 5-\mathrm{kW}$ three phases induction motor. The faults are obtained by drilling the bar of the squirrel cage. The stator currents acquisition is performed by a 24 bits acquisition card with $20 \mathrm{kHz}$ sampling frequency.

All the experiments are done in steady state conditions. The motors under study are fed by a PWM inverter with a fundamental frequency equals to $f_{s}=50 \mathrm{~Hz}$. The experimental setup is given by Fig. 4 .

\section{B. Proposed Approaches Performance}

1) Bearing faults detection: Figure 5 illustrates the periodograms using a sampling frequency $F_{s}=1000 \mathrm{~Hz}$, $N=2000$ samples, and a Hanning window for healthy and faulty induction motors with bearing faults. This figure shows that the FFT-based techniques suffer from a poor frequency resolution. Figure 6 shows the stator current spectrum using TLS ESPRIT approach for healthy and faulty induction motors with bearing faults. The spectral components caused by the specific faults appear in the spectrum for faulty induction motors in the neighborhood of the fundamental frequency.

A fault severity criterion value for different bearing fault degrees in induction motors is given by Fig. 7. According to this figure, FSC values based firstly on the estimation of fault frequency signature by TLS-ESPRIT or Root-MUSIC give almost the same results.

Figure 8 shows the evolution of the fault severity criterion with respect to sample numbers. It can be shown that for low values of samples numbers the criterion increases. This is normal since we can assume that the estimation is not optimal. For $N$ greater than 500 samples the criterion is constant for a given fault severity. we can conclude that for $N=500$ samples, the proposed approach results are reliable.

2) Broken rotor bar faults detection: Figure 9 gives the periodograms using a sampling frequency $F_{s}=1000 \mathrm{~Hz}$, $N=4096$ samples, and a Hanning window for a healthy and faulty induction motors with 3 broken rotor bar. This figure shows again that the FFT-based techniques suffer from a poor frequency resolution. Figure 10 shows the stator current spectrum by using TLS ESPRIT approach for healthy and faulty induction motors with 3 broken rotor bars. The spectral components caused by the specific faults appear in 


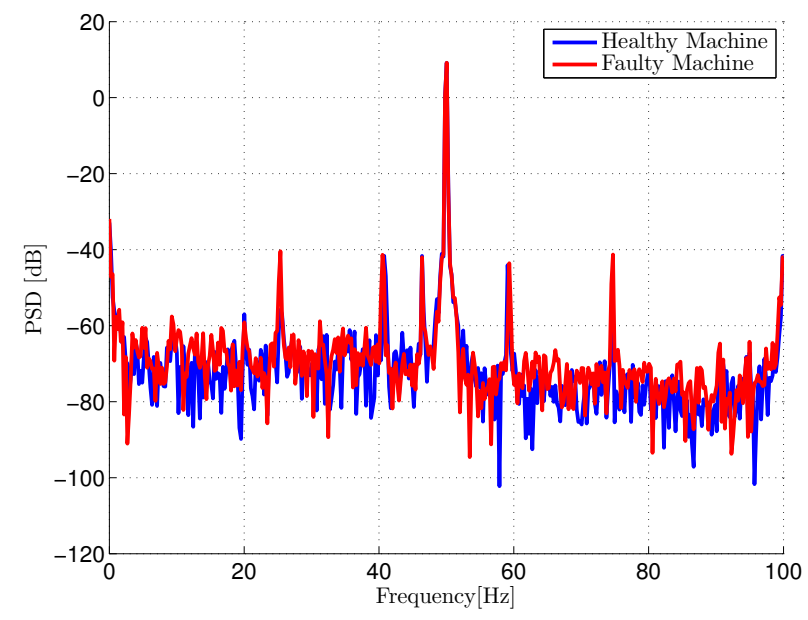

Fig. 5. Spectral analysis by Welch periodogram using Hanning window $(N=$ 2000 ) for a healthy and faulty induction motors with bearing faults.

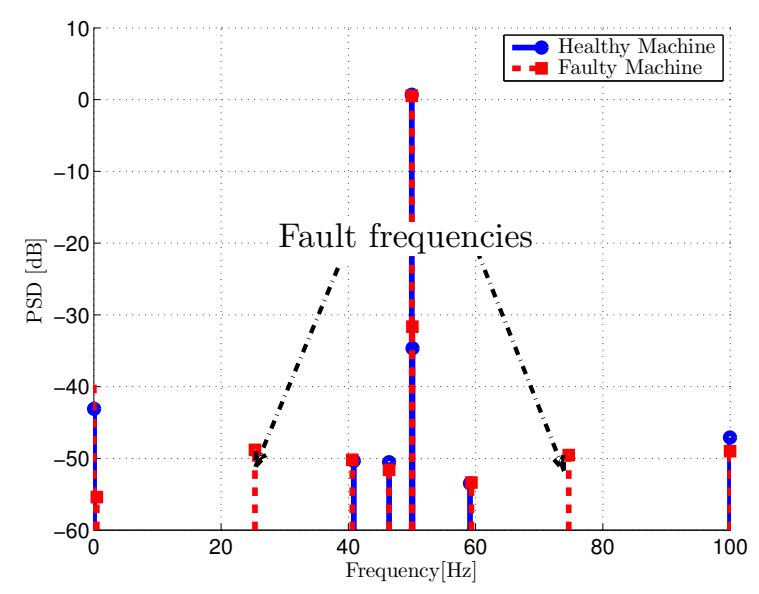

Fig. 6. Stator current spectrum based on the TLS-ESPRIT and BIC criterion for a healthy and faulty induction motors with bearing faults.

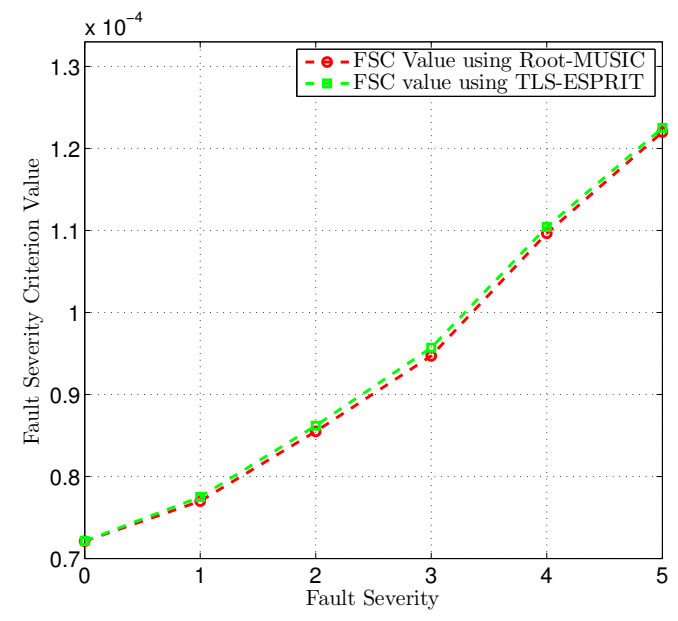

Fig. 7. Fault severity criterion value for different bearing fault degrees in induction motors.

the spectrum for faulty induction motor. The appearance of new frequencies in the spectrum is a signature of broken rotor

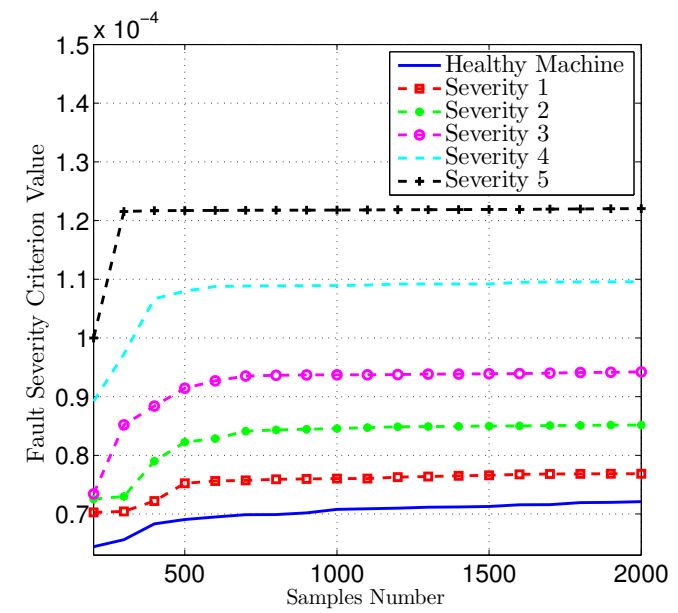

Fig. 8. Fault severity criterion value for different $N$ (samples number) for bearing faults using TLS ESPRIT, for healthy and faulty induction motors with bearing faults.

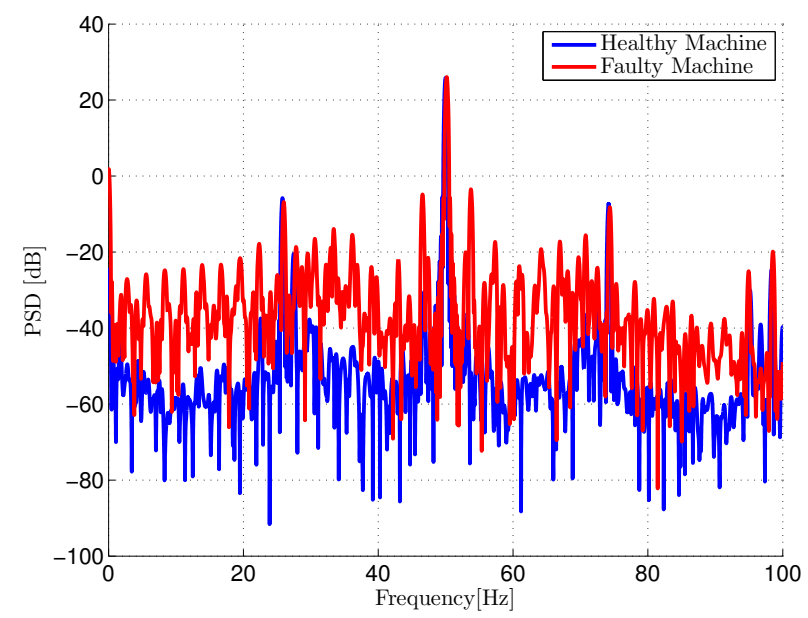

Fig. 9. Spectral analysis by Welch periodogram using Hanning window $(N=$ 4096 ) for a healthy and faulty induction motors with broken rotor bar faults.

bars. In addition, their amplitudes indicate the fault severity.

Figure 11 depicts the evolution of the proposed criterion for 1,2 , and 3 broken rotor bars. This figure clearly show that the proposed criterion allows measuring the fault severity. The behavior of the fault severity criterion value is the same for different number of broken rotor bars.

Figure 12 shows the FSC for different $N$ value using TLS ESPRIT, for healthy and faulty induction motors with broken rotor bars. For faulty motors, the FSC value increases with samples number and also with the the number of broken rotor bars (Fig 11).

\section{CONCLUSION}

This paper has proposed an induction machines condition monitoring and fault detection architecture based on advanced signal processing and statistical analysis techniques that exploits stator current measurements. In this context, a four steps process is needed: model order selection, frequency estimation, amplitude estimation, fault severity criterion value computation. We have shown that the Bayesian information 


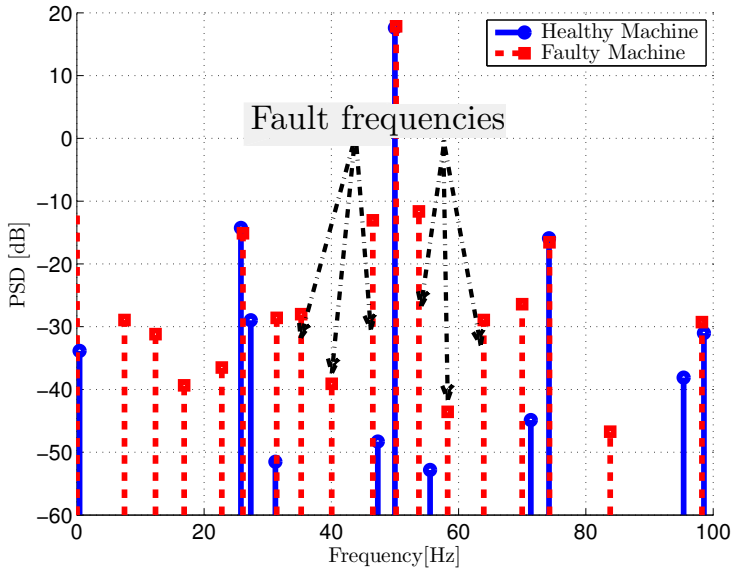

Fig. 10. Stator current spectrum based on the TLS-ESPRIT and BIC criterion for a healthy and faulty induction motors with broken rotor bars.

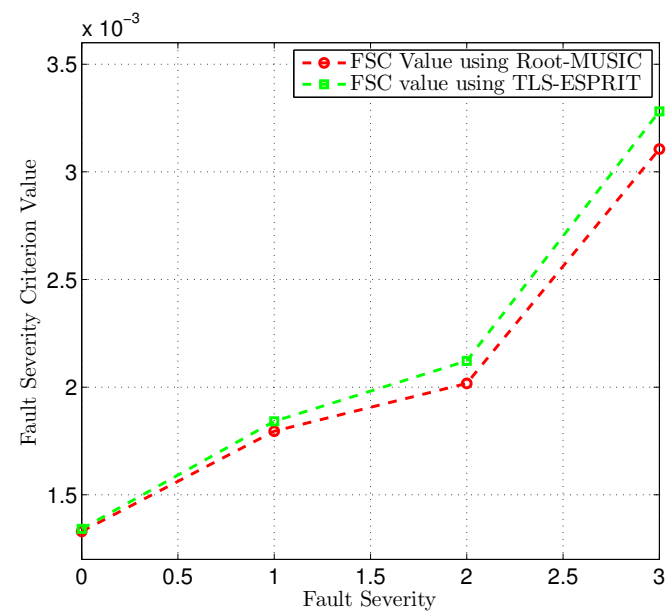

Fig. 11. Fault severity criterion for different broken rotor bars. In this figure, 0 corresponds to the healthy induction motor and the other values correspond to broken rotor bars number.

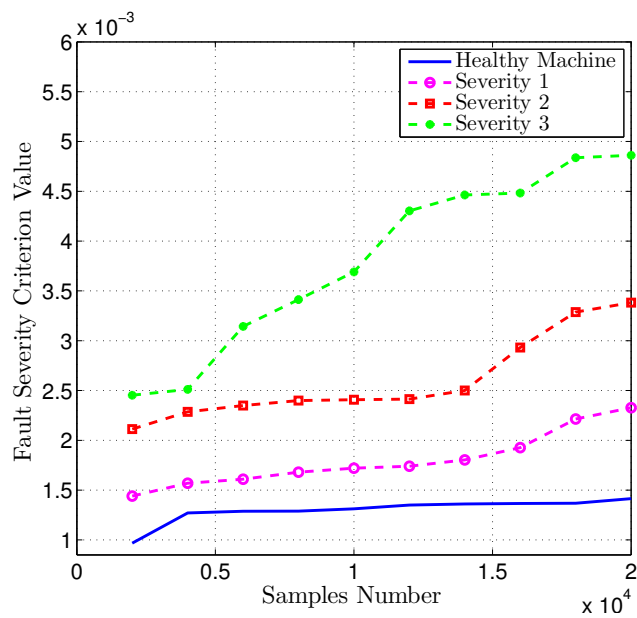

Fig. 12. Fault severity criterion value for different $N$ (samples number) for bearing faults using TLS ESPRIT, for healthy and faulty induction motors with broken rotor bars. criterion is more efficient than other information criteria. The frequencies estimated by subspace techniques are exploited for fault signature detection. The amplitudes of frequency components determined by least squares estimator indicate the severity degree of the considered faults by using the proposed fault severity criterion. According to the achieved experimental results, it can be concluded that the proposed architecture is able to efficiently and cost-effectively detect electrical and mechanical faults using stator current processing. Further works must be conducted in order to examine the feasibility of the proposed induction machines condition monitoring and fault detection architecture in case of non-stationnary environments such as renewable energy systems.

\section{REFERENCES}

[1] E. Elbouchikhi, V. Choqueuse, and M. E. H. Benbouzid, "Current frequency spectral subtraction and its contribution to induction machines bearings condition monitoring," IEEE Trans. Energy Conversion, vol. 28, no. 1, pp. 135-144, December 2013.

[2] P. Zhang, Y. Du, T. G. Habetler, and B. Lu, "A survey of condition monitoring and protection methods for medium-voltage induction motors," IEEE Trans. Ind. Appl., vol. 47, no. 1, pp. 34-46, January/ February 2011.

[3] M. Blödt, D. Bonacci, J. Regnier, M. Chabert, and J. Faucher, "On-line monitoring of mechanical faults in variable-speed induction motor drives using the wigner distribution," IEEE Trans. Ind. Electron., vol. 55, no. 2, pp. 522-533, February 2008.

[4] E. Elbouchikhi, V. Choqueuse, M. E. H. Benbouzid, and J.-F. Charpentier, "Induction machine fault detection enhancement using a stator current high resolution spectrum," in Proceedings of the 2012 IEEE IECON, pp. 3913-3918, October 2012.

[5] H. A. Toliyat, S. Nandi, S. Choi, and H. Meshgin-Kelk, Electric Machines: Modeling, Condition Monitoring, and Fault Diagnosis. CRC Press, October 2012.

[6] F. Immovilli, A. Bellini, R. Rubini, and C. Tassoni, "Diagnosis of bearing faults in induction machines by vibration or current signals: A critical comparison," IEEE Trans. Ind. Appl., vol. 46, no. 4, pp. 13501359, May 2010.

[7] L. Frosini and E. Bassi, "Stator current and motor efficiency as indicators for different types of bearing faults in induction motors," IEEE Trans. Ind. Electron., vol. 57, no. 1, pp. 244-251, January 2010.

[8] V. Choqueuse, M. E. H. Benbouzid, Y. Amirat, and S. Turri, "Diagnosis of three-phase electrical machines using multidimensional demodulation techniques," IEEE Trans. Ind. Electron., vol. 59, no. 4, pp. 2014-2023, April 2012.

[9] M. E. H. Benbouzid, "A review of induction motors signature analysis as a medium for faults detection," IEEE Trans. Ind. Electron., vol. 47, no. 5, pp. 984-993, October 2000.

[10] M. E. H. Benbouzid and G. B. Kliman, "What stator current processingbased technique to use for induction motor rotor faults diagnosis?," IEEE Trans. Energy Conversion, vol. 18, no. 2, pp. 238-244, June 2003.

[11] S. Nandi, H. A. Toliyat, and X. Li, "Condition monitoring and fault diagnosis of electrical motors-a review," IEEE Trans. Energy Conversion, vol. 20, no. 4, pp. 719-729, December 2005.

[12] J. Cusido, L. Romeral, J. Ortega, J. Rosero, and A. G. Espinosa, "Fault detection in induction machines using power spectral density in wavelet decomposition," IEEE Trans. Ind. Electron., vol. 55, no. 2, pp. 633-643, February 2008.

[13] B. Ayhan, H. J. Trussell, M.-Y. Chow, and M.-H. Song, "On the use of a lower sampling rate for broken rotor bar detection with $\mathrm{dtft}$ and ar-based spectrum methods," IEEE Trans. Ind. Electron., vol. 55, no. 3, pp. 1421-1434, March 2008.

[14] E. Elbouchikhi, V. Choqueuse, Y. Trachi, and M. Benbouzid, "Induction machine bearing faults detection based on hilbert-huang transform," in Proceedings of the 2015 IEEE ISIE, pp. 843-848, June 2015.

[15] P. Stoica and R. L. Moses, Spectral Analysis of Signals. Pearson/Prentice Hall Upper Saddle River, NJ, March 2005.

[16] S. M. Kay, Fundamentals of Statistical Processing, Volume I: Estimation Theory. Upper Saddle River, Prentice Hall, Signal Processing, April 1993. 
[17] E. Elbouchikhi, V. Choqueuse, and M. E. H. Benbouzid, "A parametric spectral estimator for faults detection in induction machines," in Proceedings of the 2013 IEEE IECON, pp. 7358-7363, November 2013.

[18] E. Elbouchikhi, V. Choqueuse, and M. E. H. Benbouzid, "Induction machine faults detection using stator current parametric spectral estimation," Mechanical Systems and Signal Processing, vol. 52, pp. 447-464, June 2014.

[19] Y. Trachi, E. Elbouchikhi, V. Choqueuse, and M. E. H. Benbouzid, "Stator current analysis by subspace methods for fault detection in induction machines," in Proceedings of the 2015 IEEE IECON, pp. 1-6, November 2012.

[20] M. E. H. Benbouzid, M. Vieira, and C. Theys, "Induction motors" faults detection and localization using stator current advanced signal processing techniques," IEEE Trans. Power Electron., vol. 14, no. 1, pp. 14-22, January 1999.

[21] Y.-H. Kim, Y.-W. Youn, D.-H. Hwang, J.-H. Sun, and D.-S. Kang, "High-resolution parameter estimation method to identify broken rotor bar faults in induction motors," IEEE Trans. Ind. Electron., vol. 60, no. 9, pp. 4103-4117, September 2013.

[22] F. Cupertino, E. De Vanna, L. Salvatore, and S. Stasi, "Analysis techniques for detection of im broken rotor bars after supply disconnection," IEEE Trans. Ind. Appl., vol. 40, no. 2, pp. 526-533, March/April 2004.

[23] M. Sahraoui, A. J. M. Cardoso, and A. Ghoggal, "The use of a modified prony method to track the broken rotor bar characteristic frequencies and amplitudes in three-phase induction motors," IEEE Trans. Ind. Appl., vol. 51, no. 3, pp. 2136-2147, May/June 2015.

[24] B. Xu, L. Sun, L. Xu, and G. Xu, "Improvement of the Hilbert method via ESPRIT for detecting rotor fault in induction motors at low slip," IEEE Trans. Energy Conversion, vol. 28, no. 1, pp. 225-233, March 2013.

[25] B. Xu, L. Sun, L. Xu, and G. Xu, "An ESPRIT-SAA-based detection method for broken rotor bar fault in induction motors," IEEE Trans. Energy Conversion, vol. 27, no. 3, pp. 654-660, September 2012.

[26] A. Garcia-Perez, R. de Jesus Romero-Troncoso, E. Cabal-Yepez, and R. A. Osornio-Rios, "The application of high-resolution spectral analysis for identifying multiple combined faults in induction motors," IEEE Trans. Ind. Electron., vol. 58, no. 5, pp. 2002-2010, May 2011.

[27] R. Romero-Troncoso, A. Garcia-Perez, D. Morinigo-Sotelo, O. DuquePerez, R. Osornio-Rios, and M. Ibarra-Manzano, "Rotor unbalance and broken rotor bar detection in inverter-fed induction motors at start-up and steady-state regimes by high-resolution spectral analysis," Electric Power Systems Research, vol. 133, pp. 142-148, April 2016.

[28] A. Garcia-Perez, R. J. Romero-Troncoso, E. Cabal-Yepez, R. OsornioRios, J. de Jesus Rangel-Magdaleno, H. Miranda, et al., "Startup current analysis of incipient broken rotor bar in induction motors using highresolution spectral analysis," in Proceedings of the 2011 IEEE ISIE, pp. 657-663, September 2011.

[29] S. H. Kia, H. Henao, and G.-A. Capolino, "A high-resolution frequency estimation method for three-phase induction machine fault detection," IEEE Trans. Ind. Electron., vol. 54, no. 4, pp. 2305-2314, August 2007.

[30] E. Radoi and A. Quinquis, "A new method for estimating the number of harmonic components in noise with application in high resolution radar," EURASIP Journal on Advances in Signal Processing, vol. 2004, no. 8, pp. 1-12, 2004.

[31] M. Wax and T. Kailath, "Detection of signals by information theoretic criteria," IEEE Trans. Acoust., Speech, Signal Processing, vol. 33, no. 2, pp. 387-392, April 1985.

[32] R. Romero-Troncoso, D. Morinigo-Sotelo, O. Duque-Perez, P. GardelSotomayor, R. Osornio-Rios, and A. Garcia-Perez, "Early broken rotor bar detection techniques in vsd-fed induction motors at steady-state," in IEEE International Symposium on Diagnostics for Electric Machines, Power Electronics and Drives (SDEMPED), pp. 105-113, IEEE, August 2013.

[33] R. Romero-Troncoso, D. Morinigo-Sotelo, O. Duque-Perez, R. OsornioRios, M. Ibarra-Manzano, and A. Garcia-Perez, "Broken rotor bar detection in vsd-fed induction motors at startup by high-resolution spectral analysis," in International Conference on Electrical Machines (ICEM), pp. 1848-1854, IEEE, September 2014.

[34] P. Stoica and Y. Selen, "Model-order selection: a review of information criterion rules," IEEE Signal Processing Mag., vol. 21, no. 4, pp. 36-47, July 2004.

[35] P. Stoica and P. Babu, "The gaussian data assumption leads to the largest Cramér-Rao bound [lecture notes]," IEEE Signal Processing Mag., vol. 28, no. 3, pp. 132-133, May 2011.

[36] M. Rosenblatt, "A central limit theorem and a strong mixing condition," Proceedings of the National Academy of Sciences of the United States of America, vol. 42, no. 1, p. 43, 1956.
[37] "International Electrotechnical Commission IEC 038, Standard Voltages," in International Electrotechnical Commission, 1999.

[38] Y. Hua, A. Gershman, and Q. Cheng, High-resolution and robust signal processing. CRC Press, 2003.

[39] G. Blanchet and M. Charbit, Digital Signal and Image Processing Using Matlab, vol. 4. Iste London, May 2006.

[40] D. G. Manolakis, V. K. Ingle, and S. M. Kogon, Statistical and Adaptive Signal Processing: Spectral Estimation, Signal Modeling, Adaptive Filtering, and Array Processing, vol. 46. Artech House Norwood, May 2005.

[41] R. Roy and T. Kailath, "ESPRIT-estimation of signal parameters via rotational invariance techniques," IEEE Trans. Acoust., Speech, Signal Processing, vol. 37, no. 7, pp. 984-995, July 1989.

[42] P. Stoica, H. Li, and J. Li, "Amplitude estimation of sinusoidal signals: survey, new results, and an application," IEEE Trans. Signal Process., vol. 48, no. 2, pp. 338-352, February 2000.

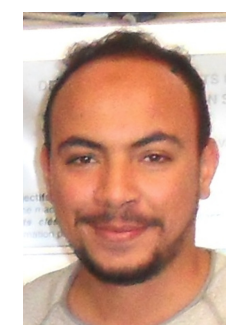

Youness Trachi was born in Morocco, in 1990. He received the M.Sc. degree in electrical engineering from the University of Lyon I, Lyon, France, in 2014. $\mathrm{He}$ is currently working toward the Ph.D. degree on condition monitoring of renewable energy systems with the University of Brest, Brest, France.

His current research interests include induction machines fault detection and diagnosis using advanced signal processing techniques.

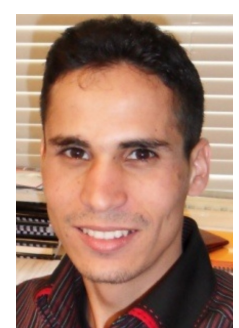

Elhoussin Elbouchikhi was born in Khemisset, Morocco, in 1987. He received the diploma engineer degree (Dipl.-Ing.) in Automatic and Electrical Engineering and Research Master's degree in Automatic Systems, Computer Science and Decision, from the National Polytechnic Institute of Toulouse (ENSEEIHT), Toulouse, France, in 2010, and the Ph.D degree in 2013 from the University of Brest, Brest, France. He has been a Post-Doctoral Researcher at ISEN Brest, Brest, France and an Associate Member of the LBMS Lab (EA 4325) from October 2013 to September 2014. Since September 2014, he is an Associate Professor at ISEN Brest, Brest, France and is an affiliated member of the Institut de Recherche Dupuy de Lôme - IRDL (FRE CNRS 3744).

His current research interests include electrical machines faults detection and diagnosis, and signal processing and statistics for power systems monitoring.

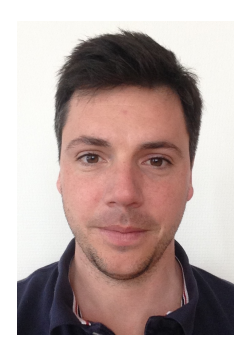

Vincent Choqueuse (S'08-M'09) was born in Brest, France, in 1981. He received the Dipl.-Ing. and the M.Sc. degrees in 2004 and 2005, respectively, from the Troyes University of Technology, Troyes, France, and the $\mathrm{PhD}$ degree in 2008 from the University of Brest, Brest, France. Since September 2009, he has been an Associate Professor with the Institut Universitaire de Technologie of Brest, University of Brest, Brest, France, and a member of the Institut de Recherche Dupuy de Lôme - IRDL (FRE CNRS 3744).

Dr. Choqueuse research interests include signal processing and statistics for power systems monitoring, Smart-Grid, digital communication and digital audio. 


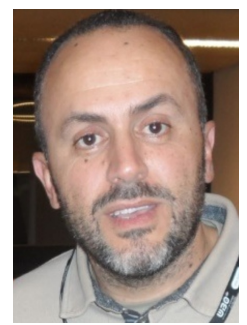

Mohamed El Hachemi Benbouzid (S'92-M'95SM'98) was born in Batna, Algeria, in 1968. He received the B.Sc. degree in electrical engineering from the University of Batna, Batna, Algeria, in 1990, the M.Sc. and Ph.D. degrees in electrical and computer engineering from the National Polytechnic Institute of Grenoble, Grenoble, France, in 1991 and 1994, respectively, and the Habilitation Diriger des Recherches degree from the University of Picardie "Jules Verne," Amiens, France, in 2000.

After receiving the Ph.D. degree, he joined the Professional Institute of Amiens, University of Picardie "Jules Verne," where he was an Associate Professor of electrical and computer engineering. Since
September 2004, he has been with the Institut Universitaire de Technologie of Brest, University of Brest, Brest, France, where he is a Professor of electrical engineering. Prof. Benbouzid is also a Distinguished Professor at the Shanghai Maritime University, Shanghai, China. His main research interests and experience include analysis, design, and control of electric machines, variable-speed drives for traction, propulsion, and renewable energy applications, and fault diagnosis of electric machines.

Prof. Benbouzid is an IEEE Senior Member. He is the Editor-in-Chief of the International Journal on Energy Conversion (IRECON). He is also an Associate Editor of the IEEE Transactions on Energy Conversion, the IEEE Transactions on Industrial Electronics, the IEEE Transactions on Sustainable Energy, and the IEEE Transactions on Vehicular Technology. 